\title{
Fast Lossless Compression of Medical Images based on Polynomial
}

\author{
Ghadah Al-Khafaji, PhD. \\ Dept. of Computer Science, Baghdad University, \\ College of Science.
}

\author{
Loay E. George, PhD. \\ Dept. of Computer Science, \\ Baghdad University, \\ College of Science
}

\begin{abstract}
In this paper, a fast lossless image compression method is introduced for compressing medical images, it is based on splitting the image blocks according to its nature along with using the polynomial approximation to decompose image signal followed by applying run length coding on the residue part of the image, which represents the error caused by applying polynomial approximation. Then, Huffman coding is applied as a last stage to encode the polynomial coefficients and run length coding. The test results indicate that the suggested method can lead to promising performance.
\end{abstract}

\section{General Terms}

Polynomial approximation within high synthetic coding architecture for lossless image compression.

\section{Keywords}

Medical images, lossless image compression, predictive coding and polynomial representation.

\section{INTRODUCTION}

Today various medical digital images currently in use such as magnetic resonance (MR), ultrasound (US), computerized tomography $(\mathrm{CT})$, nuclear medicine (NM), positron emission tomography (PET), digital subtraction angiography (DSA) and X-rays images. Lossless image compression essentially utilized for medical application that characterized by preserving the information; where the image can be reconstructed exactly as the original in which no information is lost. It is possible to do lossless compression with techniques such as Huffman coding, Arithmetic coding, Lempel-Ziv, Differential Pulse Code Modulation and Multiresolution techniques; but most of these methods leads to limited compression rate results. Reviews of medical image compression techniques can be found in [1], [2]. A comparison in performance of several different lossless techniques on various medical image types can be found in [3]-[8].

Today, there's trend in the utilization of Predictive Coding (PC) that also called Autoregressive (AR) for medical image compression where recently there's a number of researchers have exploited this technique to compress images [9]-[13] due to its simplicity, fast and easy to implement. Its implementation is generally composed of two basic steps of prediction and differentiation, in other words create an approximation image to the original one based on modelling the correlation or statistical dependency embedded between neighbouring pixels; where each pixel's value can be predicted or estimated from nearby or neighbouring pixels, and then finding the difference between the original and the predicted image one which is referred as the residual which is normally coded because of the reduced image information compared to the original.

There are many different kinds of predictive coding models depending on dependency form (causal/ acausal and the order of the model which means number of neighbours utilized) as well the structure used (1-D/2-D); for more details see [14][17]. The consideration of choosing a predictive coding model mainly depends on the tradeoff among predictor performance, the computation complexity of the predictor and the overhead parameters that implicitly affects the compression rate. In the field of medical imaging, a lot of works have been done, including [18] which showed the idea of efficient exploitation of the traditional means of predictive coding, and multiresolution predictive coding versus other lossless techniques, on a number of medical images. [19] extended the principle adopted by [20] for removing the variations between neighbouring pixels, before applying the predictive coding from fixed predictor into multiple predictors where the choice between them depending on the amount of error. [21] implies the utilization of predictive coding once or multiple times to remove the rest of the redundancy embedded between the estimated coefficients as a modified form of the work implemented by [22]. Several adaptations adopted by [23][25], to improve the predictive coding performance with such as S-Plane and Hierarchal Interpolation Techniques (HINT).

In this paper, a simple and fast lossless method for compressing medical images is introduced that based on splitting the image into non-overlapped blocks according to its nature, and utilized first order polynomial representation to remove the redundancy between neighbouring pixels that efficiently improve compression rate.

The rest of the paper is organized as follows, section 2 contains comprehensive clarification of the proposed system; the results of the proposed system is given in section 3 .

\section{THE PROPOSED SYSTEM}

The main taken concerns in the proposed system are:

- Get the benefit of varying image characteristics (details), where the image contains uniform (smooth) regions with different variation modes and edge regions; each one is compress in different way than the other and, usually deals with different number of parameters.

- Since in this paper the linear polynomial representation is adopted to remove the spatial redundancy, so three coefficients $\left(\mathrm{a}_{0}, \mathrm{a}_{1}, \mathrm{a}_{2}\right)$ are required to represent each small block. For uniform regions only the first coefficient (i.e., $\mathrm{a}_{0}$ ) is utilized; which corresponds to the mean value of the represented block. While for the blocks with significant variation trends the 3 coefficients $\left(\mathrm{a}_{0}, \mathrm{a}_{1}, \mathrm{a}_{2}\right)$ are needed. So, for 
each region it is not necessarily to use fixed number of coefficients, either 1 coefficient or 3 coefficients are used depending on the nature of the represented region.

- Run length coding and entropy encoding are used efficiently in order to minimize the bit required.

The implementation of the proposed system is explained in the following steps, the layout of the encoder is illustrated in Figure 1:

Step 1: Load the input uncompressed image $I$ of size $N \times N$

Step 2: Partition the image $(I)$ into nonoverlapped blocks of fixed size $n \times n$, such as $(4 \times 4)$ or $(8 \times 8)$ then compute the mean of each block which corresponds to $\mathrm{a}_{0}$

$a_{0}=\frac{1}{n \times n} \sum_{i=0}^{n-1} \sum_{j=0}^{n-1} I(i, j) \ldots \ldots \ldots \ldots . .(1)$

Step 3: Split the image blocks into uniform and non-uniform regions depending on mean threshold value. For uniform blocks use $\mathrm{a}_{0}$ coefficients; for non-uniform blocks use the polynomial representation approximation and find $\mathrm{a}_{1}$ and $\mathrm{a}_{2}$ coefficients according to equations $(2,3)[26]$ :

$a_{1}=\frac{\sum_{i=0}^{n-1} \sum_{j=0}^{n-1} I(i, j) \times\left(j-x_{c}\right)}{\sum_{i=0}^{n-1} \sum_{j=0}^{n-1}\left(j-x_{c}\right)^{2}} \ldots \ldots \ldots \ldots . .(2)$

$a_{2}=\frac{\sum_{i=0}^{n-1} \sum_{j=0}^{n-1} I(i, j) \times\left(i-y_{c}\right)}{\sum_{i=0}^{n-1} \sum_{j=0}^{n-1}\left(i-y_{c}\right)^{2}} \ldots \ldots .(3)$

Where $I(i, j)$ is the original image block of size $(n \times n)$ and

$x c=y c=\frac{n-1}{2}$

Step 4: Determine the approximated image value $\tilde{I}$ using the estimated polynomial coefficients for each encoded block representation:

$\tilde{I}=a_{0}+a_{1}\left(j-x_{c}\right)+a_{2}\left(i-y_{c}\right) \ldots . .(5)$

Step 5: Find the residual or prediction error as difference between the original I and the predicted one $\widetilde{I}$.

$R(i, j)=I(i, j)-\widetilde{I}(i, j) \ldots \ldots . .(6)$

Step 6: Apply Run Length Coding techniques to encode the residual image values that characterized by less correlation with smaller variance than the original pixel values, and with highly packing information around the zero. The run length code is passed through huffman coding to remove the rest of redundancy.
To reconstruct the decompressed image all the above mentioned steps are reversed as shown in Figure 2, where the decoder exploits the information received from the encoder to reconstruct the image, by first utilizing the polynomial coefficients to build a predicted image, and then adding the residual to the prediction, such that:

$I(i, j)=R(i, j)+\tilde{I}(i, j) \ldots \ldots .(7)$

\section{EXPERIMENTS AND RESULTS}

For testing the proposed system performance; it is applied on a number of medical images of different types (see Figure 3 for an over view), all the images are gray of 256 gray levels (8bits/pixel) but with different sizes. The tests have been performed using two different block sizes $\{4 \times 4$ and $8 \times 8\}$ the mean threshold value was selected depending on the image nature (feature).

The compression ratio, which is the ratio of the original image size to the compressed size, was adopted as a packing measure. Since, there is no degradation need to be evaluated in lossless compression where the decoded compressed image is identical to the original image, so the only guide here to the efficiency of proposed system is compression efficiency.

The compression rate of the proposed system is affected by two factors; first the mean threshold value that used to classify the blocks into smooth or non-smooth region whereas small value selected large number of blocks needs to have fully polynomial representation (i.e., 3 coefficients) implicitly increasing the size of the compressed information while as the mean threshold value gets bigger less number of blocks needs full polynomial representation and simply they used one coefficient representation (i.e., only $\mathrm{a}_{0}$ ); this will implicitly decrease the size of compressed information. The second factor is the size of the block whereas the block size increase the compression rate improves because less coefficient parameters required, but on the other hand the residual size increase due to insufficient model flexibility (i.e., not fitted well ).

The experimental results listed in Table 1, the feasible performance of the proposed simple lossless method on medical images, high compression is attained with fast implementation.

\section{ACKNOWLEDGMENTS}

Our thanks to the experts who have contributed towards development of the template. 
Table 1. Compression performance of the proposed system on the tested medical images

\begin{tabular}{|c|c|c|c|c|c|}
\hline \multicolumn{2}{|c|}{} & \multicolumn{2}{|c|}{ Block Size 4 } & \multicolumn{2}{c|}{ Block Size 8 } \\
\hline Tested & $\begin{array}{c}\text { Size in bytes of } \\
\text { Original image }\end{array}$ & $\begin{array}{c}\text { Size in bytes compressed } \\
\text { information }\end{array}$ & Comp.Ratio & $\begin{array}{c}\text { Size in bytes compressed } \\
\text { information }\end{array}$ & Comp.Ratio \\
\hline Brain-1 MR & 65536 & 9796 & 6.6901 & 6800 & 9.6376 \\
\hline Brain-2 MR & 65536 & 10202 & 6.4238 & 6996 & 9.3676 \\
\hline Brain-3 MR & 40000 & 7392 & 5.4113 & 5238 & 7.6365 \\
\hline Knee-1 MR & 65536 & 10156 & 6.4529 & 7008 & 9.3516 \\
\hline Knee-2 MR & 14400 & 2578 & 5.5857 & 1754 & 8.2098 \\
\hline Knee-3 MR & 262144 & 49934 & 5.2498 & 34814 & 7.5298 \\
\hline echo & 65536 & 11104 & 5.9020 & 7890 & 8.3062 \\
\hline Chest X-ray & 65536 & 11132 & 5.8872 & 7368 & 8.8947 \\
\hline
\end{tabular}

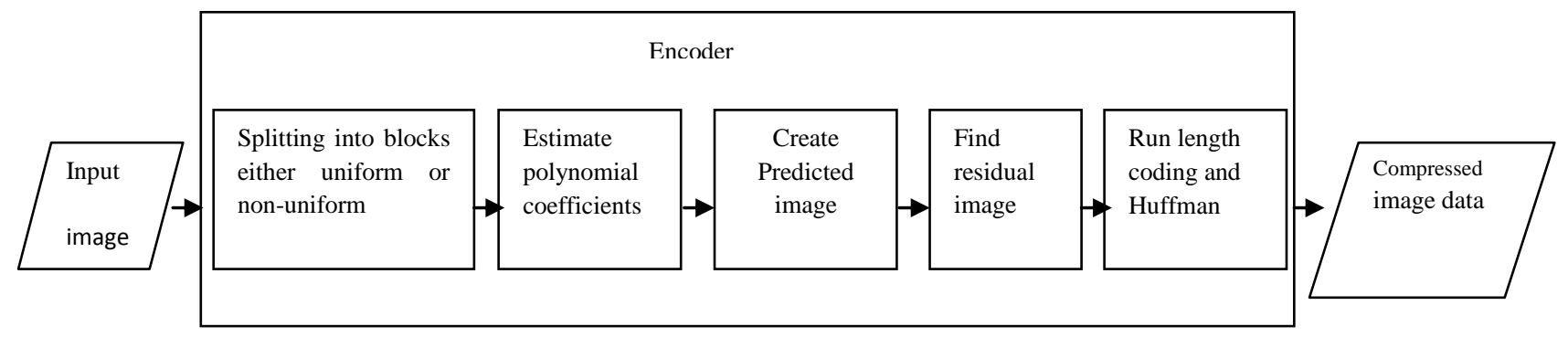

Fig 1: Encoder structure of the proposed system

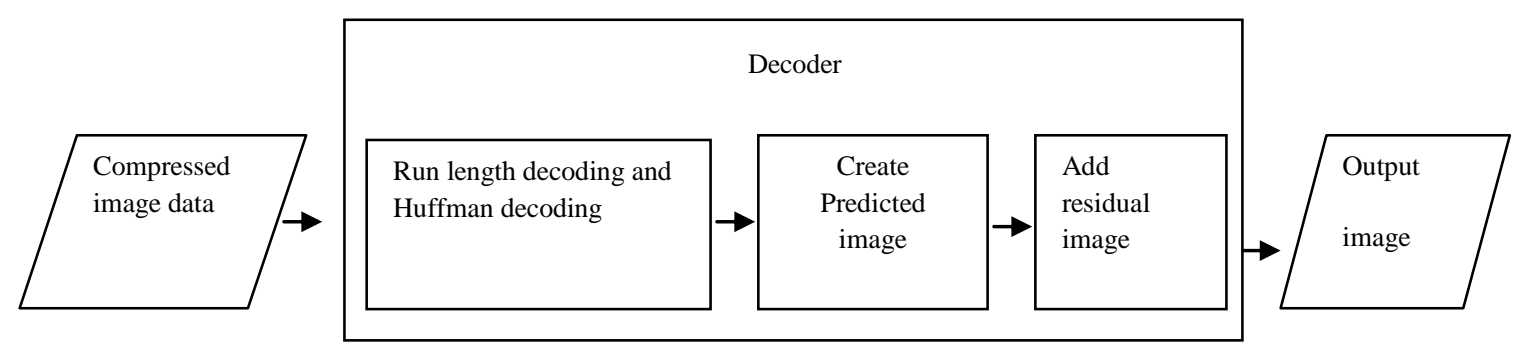

Fig 2: Decoder structure of the proposed system 


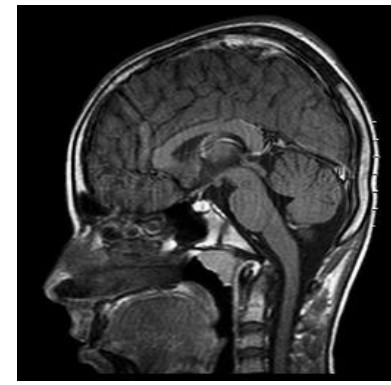

Brain-1 MR 256×256

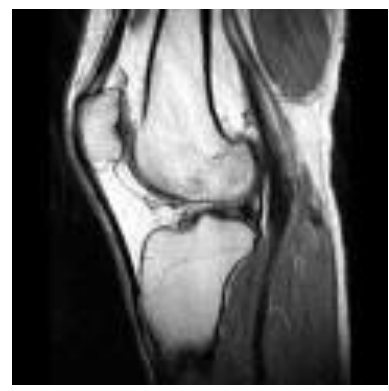

Knee-2 MR $120 \times 120$

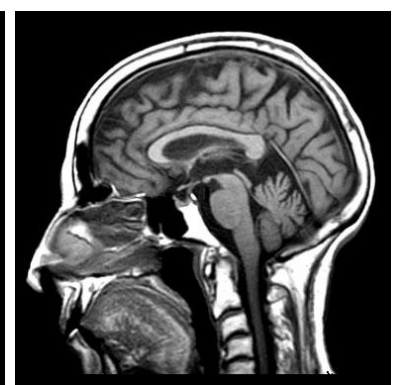

Brain-2 MR 256×256

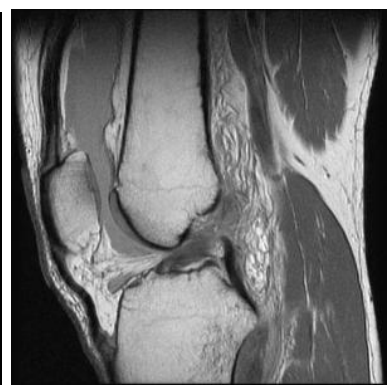

Knee-3 MR 512×512

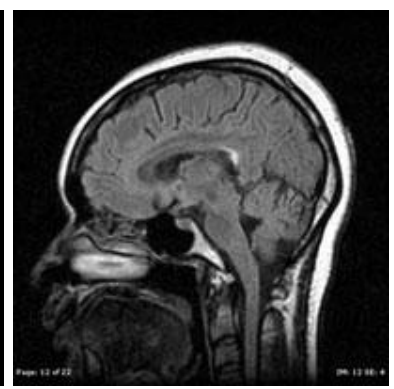

Brain-3 MR 200×200



Echo-US 256×256

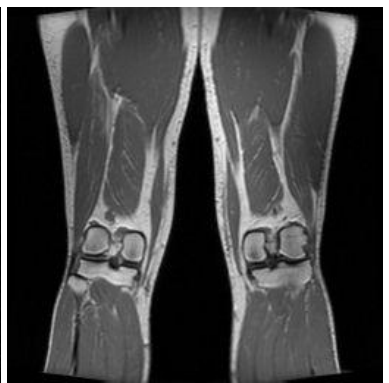

Knee-1 MR 256×256

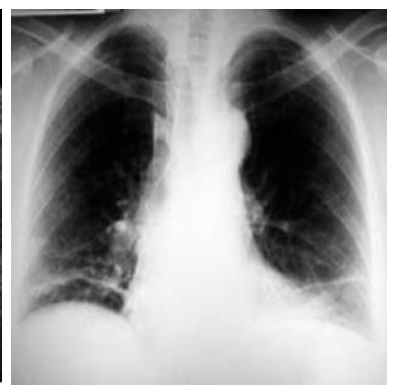

Chest X-ray $256 \times 256$

Fig 3: Overview of the medical test images

\section{REFERENCES}

[1] Bramble, J. M., Huang, H. K. and Murphy, M. D. 1988. Image Data Compression. Radiology, 23, 707-712.

[2] Wong, S., Zaremba, L., Gooden D. and Huang, H. K. 1995. Radiologic Image Compression - A Review. Proceedings of the IEEE, 83(2), 194-218

[3] Roos, P., Viergever M.A., Duke, M. C. A. and Peters, J. H. 1988. Reversible Intraframe Compression of Medical Images. IEEE Transactions on Medical Imaging, 7(4), 328-336.

[4] Bramble, J. M. 1989. Comparison of Information Preserving and Information-Losing Data-Compression Algorithms for CT Images. Radiology, 170, 543-455.

[5] Heer, V. K. and Reinfelder, H.E. 1990. A Comparison of Reversible Methods for Data Compression. SPIE Medical Imaging, 1233, 254-365.

[6] Ramabadran, T. V. and Chen, K. 1992. The Use of Contextual Information in the Reversible Compression of Medical Images. IEEE Transactions on Medical Imaging, 11(2), 185-195.

[7] Viergever, M. A. and Roos, P. 1993. Hierarchical Interpolation. IEEE Engineering in Medicine and Biology Magazine, 12(1), 48- 55.

[8] Miaou, S-G., Ke, F-S. and Chen, S-C. 2009. A Lossless Compression Method for Medical Image Sequences

[15] Connor, D. J., Pease, R. F. W. and Scholes, W. G. 1971. Television Coding using Two-Dimensional Spatial Prediction. Bell System Technology Journal, 50(3), 1049-1061.
Using JPEG-LS and Interframe Coding. IEEE Transactions on Information Technology in Biomedicine, 13(5), 818-821.

[9] Das, M., Tan, S.Y. and Loh, N. K. 1987. Adaptive Predictive Coding of Images Based upon Multiplicative Time Series Modeling, Proceeding of the IEEE International Conference on Acoustic, Speech and Signal Processing, 1386-1389.

[10] Salo, J. and Neuvo, Y. 1988. A New Two Dimensional Predictor Design for DPCM Coding of Video Signals. Signal Processing of HDTV, 207-211.

[11] Das, M. and Loh, N. K. 1990. New Studies on Adaptive Coding of Images using Multiplicative Autoregressive Models. 10th IEEE Region Conference on Communication, 442-446.

[12] Takamura, S. 1996. Efficient Lossless Still Image Coding Based on the Autoregressive Model. Ph.D. thesis, University of Tokyo.

[13] Chakravarti, S., Jung, T-P., Ahalt, S. C. and Krishnamurthy, A. K. 1999. Comparison of Prediction Methods for Differential Image Processing Applications, Proceedings of the IEEE International Conference on System Engineering, 210-213.

[14] Harrison, C. W. 1952. Experiments with Linear Prediction in Television. Bell System Technology Journal, 31, 764-783.

[16] Musmann, H. G. 1979. Predictive Image Coding. Image Transmission Techniques Advances in Electronics and Electron Physics, 12, 73-112.

[17] Balram, N. and Moura, J. M. F. 1996. Noncausal Predictive Image Codec. IEEE Transactions on Image Processing, 5(8), 1229-1242. 
[18] Das, M. and Burgett, S. 1993. Lossless Compression of Medical Images Using Two Dimensional Multiplicative Autoregressive Models. IEEE Transactions on Medical Imaging, 12(4), 721-726.

[19] Chen, Z. D., Chang, R. F. and Kuo, W. J., 1999. Adaptive Predictive Multiplicative Autoregressive Model for Medical Image Compression. IEEE Transactions on Medical Imaging, 18(2), 181-184.

[20] Das, M. and Burgett, S. 1992. A Lossless Medical Image Compression Scheme Based on Two-Dimensional Space Varying Multiplicative Autoregressive Models. Technical Report TR-92-ESE-06-02, University of Oakland.

[21] Das, M. and Lin, C. 1996. Lossless Compression of Medical Images Using Hierarchical Autoregressive Models. 9th IEEE Symposium on Computer-Based Medical Systems, 6-11.
[22] Kakusho, O. and Yanagida, M. 1982. Hierarchical AR Model for Time Verying Speech Signals. Proceedings of the IEEE international conference on Acoustics, Speech and Signal Processing, 1295-1298.

[23] Tang, H. and Kamata, S-I. 2006. A Gradient Based Predictive Coding for Lossless Image Compression. IEICE Transactions on Information and Systems, E89D(7), 2250-2256.

[24] Avramović, A. and Savić, S. 2011. Lossless Predictive Compression of Medical Images. Serbian Journal of Electrical Engineering, 8(1), 27-36.

[25] Mukherjee, A., Sarkar, M. and Halder, A. 2012. Predictive Lossless Color Image Compression using Arithmetic Operation. International Journal of Computer Applications, 43(5), 43-46.

[26] George, L. E. and Sultan, B. 2011. Image Compression Based on Wavelet, Polynomial and Quadtree. Journal of Applied Computer Science \& Mathematics, 11(5), 15-20. 